\title{
Donor-Acceptor Pair Luminescence of P-Al and N-Al Pairs in 3C-SiC and the Ionization Energy of the $\mathbf{P}$ Donor
}

\author{
Ivan Ivanov, Anne Henry, Fei Yan, Wolfgang J. Choyke and Erik Janzén
}

\section{Linköping University Post Print}

N.B.: When citing this work, cite the original article.

Original Publication:

Ivan Ivanov, Anne Henry, Fei Yan, Wolfgang J. Choyke and Erik Janzén, Donor-Acceptor Pair Luminescence of P-Al and N-Al Pairs in 3C-SiC and the Ionization Energy of the P Donor, 2011, Materials Science Forum Vols. 679-680, 245-248.

http://dx.doi.org/10.4028/www.scientific.net/MSF.679-680.245

Copyright: Trans Tech Publications 


\title{
Donor-Acceptor Pair Luminescence of P-Al and N-Al Pairs in 3C-SiC and the Ionization Energy of the P Donor
}

\author{
I.G. Ivanov ${ }^{1, a}$, A. Henry ${ }^{1, b}$, Fei Yan ${ }^{2, c}$, W.J. Choyke ${ }^{2, d}$, and E. Janzén ${ }^{1, e}$ \\ ${ }^{1}$ Linköping University, Department of Physics, Chemistry and Biology, 58183 Linköping, Sweden \\ ${ }^{2}$ University of Pittsburgh, Physics \& Astronomy Department, 100 Allen Hall 3941 O'Hara Street, \\ Pittsburgh, PA, 15260 USA \\ aiiv@ifm.liu.se, ${ }^{\mathrm{b}}$ ahy@ifm.liu.se, ${ }^{\mathrm{c}}$ fey6@pitt.edu, ${ }^{\mathrm{d}}$ choyke@pitt.edu, erj@ifm.liu.se
}

Keywords: phosphorus donor, ionization energy, donor-acceptor pair luminescence

\begin{abstract}
The analysis of the donor-acceptor pair luminescence of $\mathrm{P}-\mathrm{Al}$ and $\mathrm{N}-\mathrm{Al}$ pairs obtained recently for the cubic $3 \mathrm{C}$ polytype of $\mathrm{SiC}$ is viewed in some detail. A detailed consideration is given to the fitting procedure applied to the $\mathrm{P}-\mathrm{Al}$ and $\mathrm{N}-\mathrm{Al}$ spectra. Fit with theoretical models of spectra of type I and type II are applied to both $\mathrm{N}-\mathrm{Al}$ and $\mathrm{P}-\mathrm{Al}$ experimental spectra, and it is demonstrated that only contribution from $\mathrm{P}$ on $\mathrm{Si}$ site is observable in the presented samples. The accuracy of the obtained phosphorus ionization energy of $48.1 \mathrm{meV}$ is also discussed.
\end{abstract}

\section{Introduction}

The cubic polytype of silicon carbide (3C-SiC) has been less studied than the hexagonal polytypes $4 \mathrm{H}$ - and $6 \mathrm{H}-\mathrm{SiC}$, probably because most of the efforts in high quality crystal growth during the past two decades have been devoted to the hexagonal polytypes, since they are more attractive for electronic applications. However, a revived interest in the growth of $3 \mathrm{C}-\mathrm{SiC}$ can be noticed in the past few years, which stipulates the need of completing the knowledge database concerning the shallow dopants in this semiconductor. Nitrogen is the common shallow donor in all $\mathrm{SiC}$ polytypes, and its ionization energy in $3 \mathrm{C}-\mathrm{SiC}$ has been determined accurately using Fouriertransform infrared (FTIR) absorption [1,2]. This work aims at the determination of the phosphorus ionization energy in 3C-SiC by comparison of the spectra of high-quality CVD-grown 3C-SiC epitaxial layers intentionally doped with $\mathrm{P}$ donors and $\mathrm{Al}$ acceptors with previously published [3] and recently re-measured spectra of $\mathrm{N}$-Al pairs in the same polytype. A fitting procedure similar to the one described in [4] is applied in order to obtain an accurate value for the ionization energy of the $\mathrm{P}$ donor, as well as information on its site occupation, and the method of fitting is described in some detail.

\section{Experimental Details and Method of Fitting}

Samples and Photoluminescence (PL) Spectra. The P-Al co-doped samples of 3C-SiC used in this study are millimetre-sized inclusions in $\sim 30 \mu \mathrm{m}$ thick epitaxial layers of $4 \mathrm{H}$ - and $6 \mathrm{H}$-SiC grown by chemical vapour deposition (CVD) with a very low $\mathrm{N}$ background doping level (see [5] for details). All samples show pronounced donor-acceptor pair luminescence and no sign of the nitrogen donor-bound exciton lines, as shown in Fig. 1. The $\mathrm{N}-\mathrm{Al}$ sample needed as a reference in this work is a single crystal Lely platelet with unintentional but rather high doping level similar to the one used in [3]. The PL spectra were excited with low power $(<10 \mathrm{~mW})$ using the $351 \mathrm{~nm}$ line of an $\mathrm{Ar}^{+}$-ion laser (325 nm from a He-Cd laser in the $\mathrm{N}$-Al case), and registered in backscattering geometry with a resolution better than $1 \AA$ using a monochromator coupled to a CCD camera.

Theoretical Method. The method for synthesizing the theoretical donor-acceptor pair (DAP) spectra is explained in detail in [6] for the case of the hexagonal $4 \mathrm{H}-\mathrm{SiC}$. However, in the case of $3 \mathrm{C}-\mathrm{SiC}$ the following simplifications take place. There are no inequivalent lattice sites and only two different lattice sites ( $\mathrm{C}$ and Si site) which can be occupied by donors or acceptors in the unit cell of 


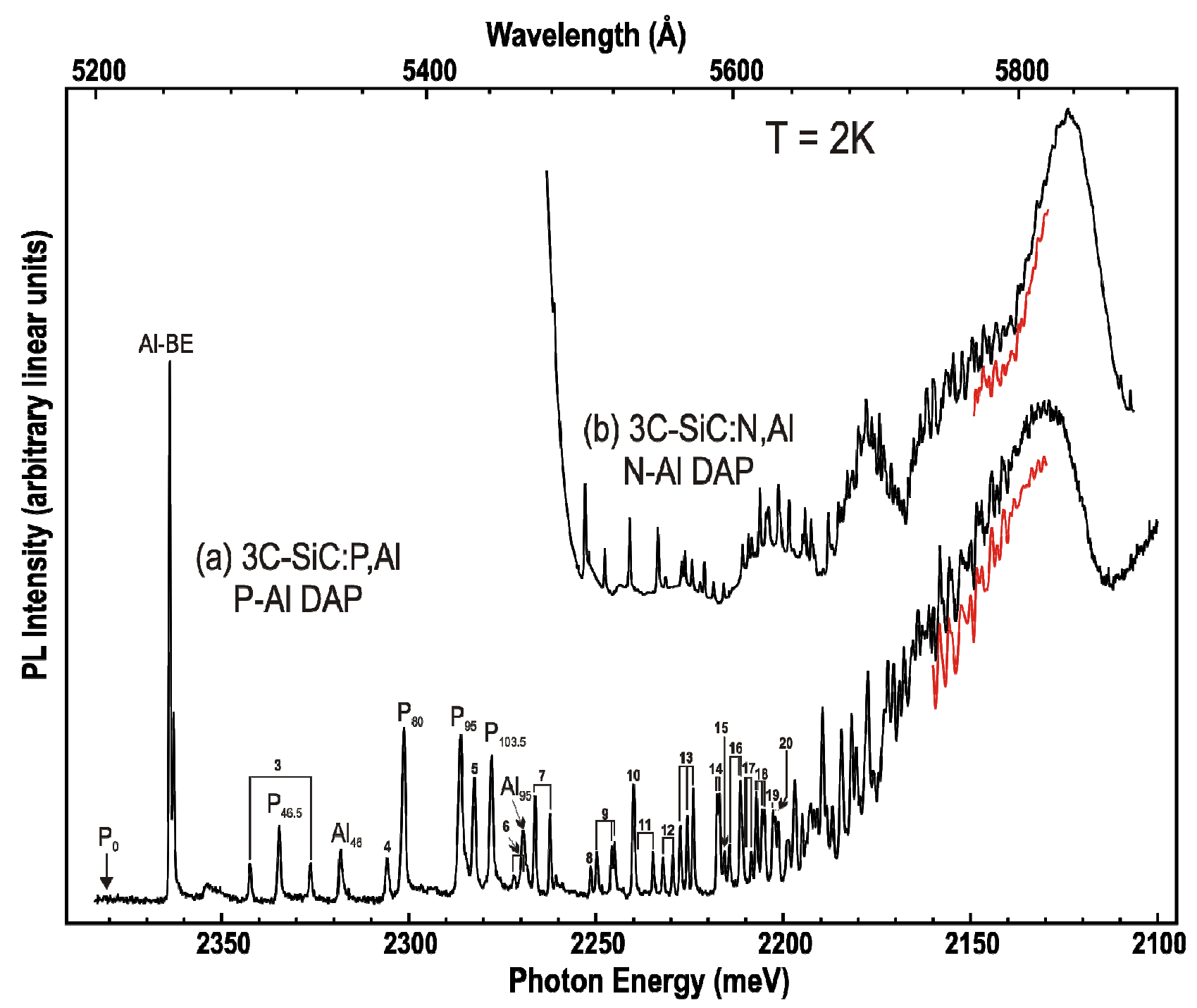

Fig. 1. Experimental spectra of 3C-SiC doped with (a) $\mathrm{P}$ and $\mathrm{Al}$, and (b) $\mathrm{N}$ and $\mathrm{Al}$. The curves slightly downshifted from the corresponding spectra for better visibility represent the best fit to the experimental spectra in the region of intermediate pairs. The limits of the fitted region are marked with the Coulomb energies according to Eq. (1) and the approximate corresponding shell numbers (m). The identification of the close-pair lines (marked with their corresponding shell numbers), many of which exhibit also splittings, is presented in detail in [5].

the cubic polytype. Hence, for substitutional dopants only spectra of type I (when the donor and the acceptor substitute the same host atom) or type II (when they substitute different host atoms) are possible. Furthermore, for the cubic lattice the dielectric constant $\varepsilon$ is isotropic, hence so is the Coulomb interaction between the ionized donor and acceptor, if all multipole corrections [7] are neglected. This is in contrast to the presence of inequivalent subshells within the shells of the hexagonal polytypes (cf. [4,6]).

The positions of the DAP lines in the spectrum are modelled according to the equation

$$
\hbar \omega_{R}=E_{g}-E_{D}-E_{A}+\frac{e^{2}}{\varepsilon R}=\hbar \omega_{\infty}+\frac{e^{2}}{\varepsilon R}
$$

where $\hbar \omega_{R}$ is the energy of a photon emitted from a donor-acceptor pair at a distance $R$ (the line position), $E_{g}, E_{D}$, and $E_{A}$ are the electronic band gap, and the donor and the acceptor ionization energies, respectively, $e$ is the electron charge, and $\hbar \omega_{\infty}=E_{g}-E_{D}-E_{A}$. Eq. (1) assumes point charges for the ionized donor and acceptor after the electron-hole recombination, and undisturbed donor and acceptor ground states before the recombination, therefore it does not describe well close 


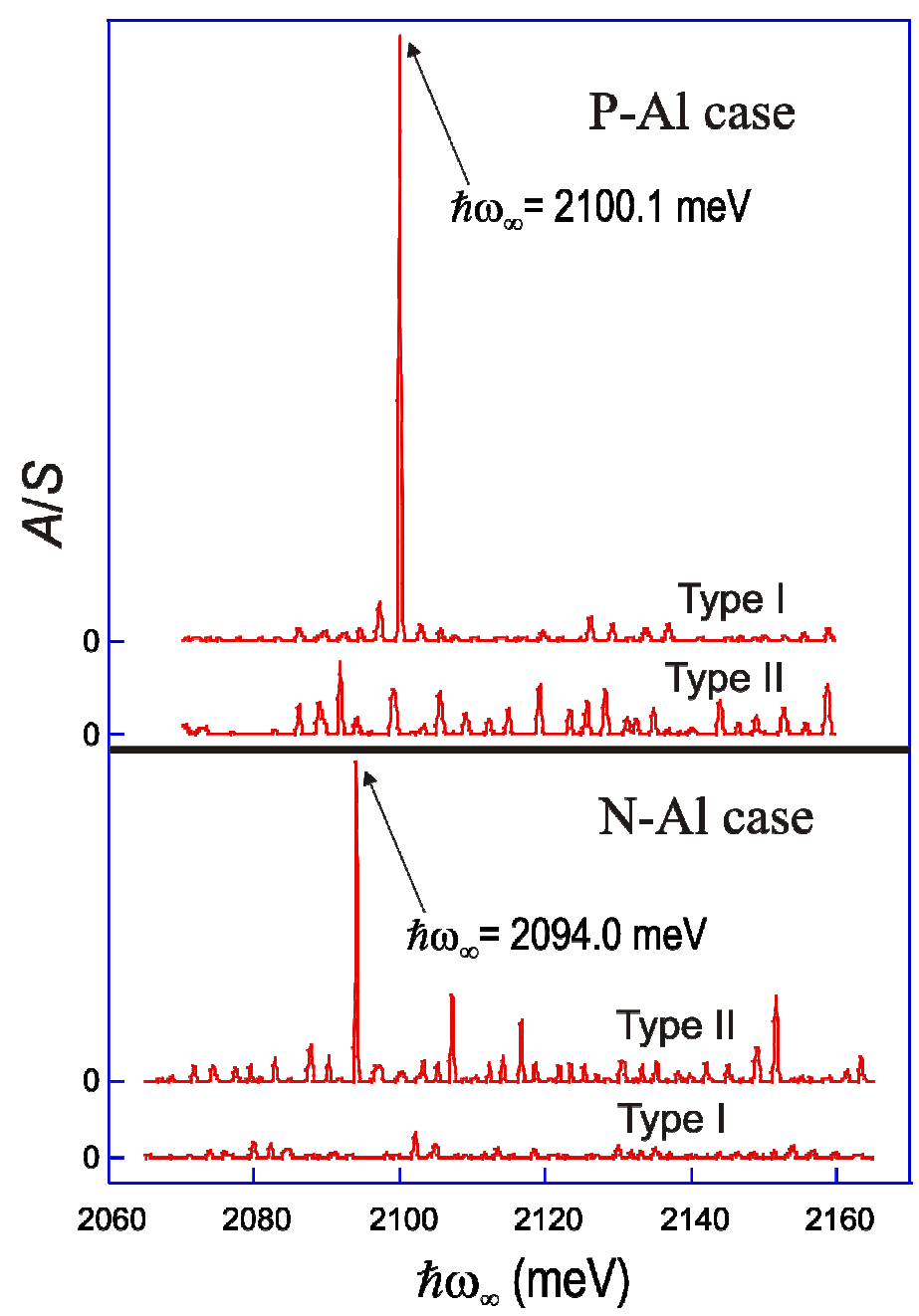

Fig. 2. Variation of the fit-quality criterion $A / S$ with $\hbar \omega_{\infty}$ for the $\mathrm{P}-\mathrm{Al}$ and $\mathrm{N}-\mathrm{Al}$ cases using theoretical spectra of type I or type II, as denoted. The four curves have the same vertical scale. pairs (small values of $R$ ). For such pairs $\hbar \omega_{R}$ depends not only on the distance $R$ but also on the orientation of the pair axis with respect to the main crystal axes, which results in line splitting of the lines associated with close pairs (see [5] for details). However, all corrections to Eq.(1) become negligible with increasing $R$ and it is shown [4-6] that a model spectrum built using Eq. (1) alone provides an accurate match to the low-energy part of the experimental spectrum corresponding to intermediate and large values of $R$.

\section{Results and Discussion}

Models of the DAP spectra of both type I and type II are built according to Eq. (1) for 3C-SiC according to the following procedure. The low-temperature value of the lattice constant $a=4.3585 \AA$ and $\varepsilon=$ 9.82 [1] are used to calculate the relative DAP-line positions. The resulting sharp lines with intensities proportional to the number of atoms in different shells corresponding to different discrete values of $R$ are replaced by Gaussians of line width $\sim 0.6 \mathrm{meV}$, chosen to be slightly larger than the spectral resolution. Then the spectrum is multiplied by a smooth exponential factor in order to account for the decreasing overlap between the electron and hole wave functions, $\exp (-$

$\mathrm{E}_{0} / \mathrm{E}_{\mathrm{C}}$ ), where $\mathrm{E}_{\mathrm{C}}=e^{2} / \varepsilon R$ is the Coulomb energy and $\mathrm{E}_{0}=120 \mathrm{meV}$ is chosen empirically. This choice is not quite arbitrary, because the factor should be of the order of $\exp \left(-R / a_{D}\right)$, where $a_{D}$ is the effective Bohr radius of the donor (assuming that the acceptor Bohr radius is much smaller). The latter exponent can easily be transformed to $\exp \left(-2 \mathrm{Ry}_{\mathrm{eff}} / \mathrm{Ec}\right)$, where $\mathrm{Ry}_{\mathrm{eff}}$ is the effective Rydberg for the donor, about $35 \mathrm{meV}$ for $3 \mathrm{C}-\mathrm{SiC}$, hence the empirical value of $\mathrm{E}_{0}=120 \mathrm{meV}$ is not very far from $2 \mathrm{Ry}_{\text {eff }}=70 \mathrm{meV}$. (It should be noted that variations of $\mathrm{E}_{0}$ in the limits $50-150 \mathrm{meV}$ do not produce visible deterioration of the fit, hence the exact value of $\mathrm{E}_{0}$ is not very significant.) Thus, theoretical spectra $\mathrm{T}\left(\mathrm{E}_{\mathrm{C}}\right)$ are obtained for both type I and type II arrangements. Finally, the experimental spectrum is fitted by the function

$$
\mathrm{Ty}(\mathrm{E})=A \mathrm{~T}\left(\mathrm{E}-\hbar \omega_{\infty}\right)+\mathrm{P}(\mathrm{E}) .
$$

Here $\mathrm{E}$ is the photon energy and $\mathrm{P}(\mathrm{E})$ is a smooth polynomial background aiming at reproducing the background in the real spectrum [5]. The polynomial coefficients and the amplitude of the theoretical curve $A$ are obtained from the least-squares method in a standard way. The quantity $\hbar \omega_{\infty}$ is the only fit parameter, which is varied in order to obtain the best fit to the experimental spectrum. Usually only a part of the simulated spectrum is used to fit the experimental one. As explained in [5], the useful part corresponding to pairs at intermediate distances lies between shells, approximately, $76 \leq m \leq 252(26.8 \AA<R<48.9 \AA)$ for the $\mathrm{N}$-Al case and to $58 \leq m \leq 239(24.5 \AA$ 
$<\mathrm{R}<49.7 \AA$ ) for the $\mathrm{P}-\mathrm{Al}$ one, where $m$ is the shell number. The criterion used to quantify the quality of the fit in the case of $3 \mathrm{C}-\mathrm{SiC}$ is simply the ratio $A / S$ (see [5] for details), where $S$ is the least-squares sum (the sum of the squares of the deviations between the experimental and theoretical points). The variation of $A / S$ with $\hbar \omega_{\infty}$ for the four fits considered here (type I and type II spectra applied to N-Al and P-Al cases) are shown in Fig. 2. Obviously, a sharp peak marks the correct value of $\hbar \omega_{\infty}$ in the corresponding fit, which can be then verified by visual inspection of the fit, as shown in Fig. 1.

As expected from the fact that $\mathrm{N}$ substitutes $\mathrm{C}$ and $\mathrm{Al}$ substitutes $\mathrm{Si}$, only the type II spectrum provides a good fit to the $\mathrm{N}-\mathrm{Al}$ pair luminescence and the value of $\hbar \omega_{\infty}(N-A l)=E_{g}-E_{D}-E_{A}=$ $2094.0 \mathrm{meV}$ obtained from the fit is very close to the value of $2093.4 \mathrm{meV}$ reported in [3]. On the other hand, the P-Al pair luminescence almost perfectly fits with the type I spectrum (see Fig. 1), thus confirming that phosphorus and aluminium atoms reside on the same (silicon) sublattice. Hence, although theoretical work [8] predicts the possibility of $\mathrm{P}$ also occupying carbon sites, we were not able to identify any $\mathrm{P}_{\mathrm{C}}-\mathrm{Al}$ contribution in our donor-acceptor pair spectra. No contribution from $\mathrm{P}_{\mathrm{C}}$ can be identified in the region of the no-phonon lines and the closest DAP lines either, since all the lines in that high-energy part of the spectrum are identified in [5] either as P- and Albound excitons, or as $\mathrm{P}_{\mathrm{Si}}-\mathrm{Al}$ close pairs, consistent with the much lower probability of forming $\mathrm{P}_{\mathrm{C}}$ than $\mathrm{P}_{\mathrm{Si}}[9]$. The value $\hbar \omega_{\infty}(P-A l)=E_{g}-E_{D}-E_{A}=2100.1 \mathrm{meV}$ is obtained from the fit of the spectrum with the sharpest lines (inclusion of $3 \mathrm{C}$ in $4 \mathrm{H}-\mathrm{SiC}$, cf. Fig. 1). This value is used to determine the ionization energy of $\mathrm{P}_{\mathrm{Si}}, E_{D}\left(\mathrm{P}_{\mathrm{Si}}\right)=48.1 \mathrm{meV}$, employing the value of $\hbar \omega_{\infty}(N-A l)$ and the known ionization energy of the $\mathrm{N}$ donor in $3 \mathrm{C}-\mathrm{SiC}, 54.2 \mathrm{meV}$ [1]. We note that $E_{D}\left(\mathrm{P}_{\mathrm{Si}}\right)$ is very close to the effective-mass theoretical donor ionization energy of $47.2 \mathrm{meV}$.

Since our value is very close also to the energy of an unidentified shallow donor (47.8 \pm 0.2 $\mathrm{meV}$ ) observed in the absorption spectra of [1,2], it is worthwhile to investigate the error bars of our measurement. Judging from the dependence of $A / S$ on $\hbar \omega_{\infty}$ displayed in Fig. 2, the error inherent to the fit is only $\pm 0.1 \mathrm{meV}$. However, we have noticed that not only the line width, but also the spectral positions of the sharp DAP lines in different samples (inclusions of 3C in different polytypes) may appear shifted by up to $1 \AA(\sim 0.4 \mathrm{meV})$. Hence, it is reasonable to set the error bar, as follows, $E_{D}\left(\mathrm{P}_{\mathrm{Si}}\right)=48.1 \pm 0.4 \mathrm{meV}$, and our value agrees with the value of [1] within the error bars. This suggests that the unidentified donor in their sample might actually be phosphorus.

In conclusion, we have observed well-structured DAP emission in 3C-SiC intentionally doped with $\mathrm{P}$ and $\mathrm{Al}$, which can be entirely explained by considering $\mathrm{P}_{\mathrm{Si}}-\mathrm{Al}_{\mathrm{Si}}$ pairs (see also [5]). Comparison of the fit of the spectra of $\mathrm{P}-\mathrm{Al}$ and $\mathrm{N}-\mathrm{Al}$ pairs yields the ionization energy of the $\mathrm{P}_{\mathrm{Si}}$ donor, $48.1 \pm 0.4 \mathrm{meV}$, using the known ionization energy of the $\mathrm{N}_{\mathrm{C}}$ donor, $54.2 \mathrm{meV}$.

Acknowledgements. Support from the Swedish Research Council and the Knut and Alice Wallenberg Foundation is gratefully acknowledged. FY and WJC thank the II-VI Foundation for partial support of their research.

\section{References}

[1] W.J. Moore, P.J. Lin-Chung, et al., Phys. Rev. B 48 (1993), p. 12289.

[2] W.J. Moore, J.A. Freitas, Jr., and P.J. Lin-Chung, Solid State Commun. 93 (1995), p. 389.

[3] W.J. Choyke and L. Patrick, Phys. Rev. B 2 (1970), p. 4959.

[4] I.G. Ivanov, A. Henry, and E. Janzén, Phys. Rev. B 71 (2005), p. 241201(R).

[5] I.G. Ivanov, A. Henry, F. Yan, W.J. Choyke, and E. Janzén, J. Appl. Phys. 108, 063532 (2010).

[6] I.G. Ivanov, A. Henry, and E. Janzén, Mater. Sci. Forum. 527-529 (2006), p. 601.

[7] L. Patrick, Phys. Rev. 180 (1969), p. 794.

[8] E. Rauls, U. Gerstmann, Th. Frauenheim, and H. Overhof, Physica B 340-342 (2003), p. 184.

[9] M. Bockstedte, A. Mattausch, and O. Pankratov, Appl. Phys. Lett. 85 (2004), p. 58. 